\title{
Attenuation of Rayleigh waves due to surface roughness ${ }^{\text {a) }}$
}

Georgios Sarris, ${ }^{1, b)}$ Stewart G. Haslinger, ${ }^{2}$ Peter Huthwaite, ${ }^{1}$ Peter B. Nagy, ${ }^{3, c)}$ and Michael J.S. Lowe ${ }^{1}$

1) Department of Mechanical Engineering, Imperial College London, London, SWr 1AY, United Kingdom

${ }^{2)}$ Department of Mathematical Sciences, University of Liverpool, Liverpool, L69 7ZL, United Kingdom

${ }^{3)}$ Department of Aerospace Engineering and Engineering Mechanics, University of Cincinnati, Cincinnati, OH 45221, USA

a) Manuscript accepted for publication in JASA - available at https://doi.org/10.1121/10.0005271 
Rayleigh waves are well known to attenuate due to scattering when they propagate over a rough surface. Theoretical investigations have derived analytical expressions linking the attenuation coefficient to statistical surface roughness parameters, namely the surface's RMS height and correlation length, and the Rayleigh wave's wavenumber. In the literature, three scattering regimes have been identified - the geometric (short wavelength), stochastic (short to medium wavelength) and Rayleigh (long wavelength) regimes. This study uses a high-fidelity two-dimensional finite element (FE) modelling scheme to validate existing predictions and to provide a unified approach to studying the problem of Rayleigh wave scattering from rough surfaces, as the same model can be used to obtain attenuation values, regardless of the scattering regime. In the Rayleigh and stochastic regimes very good agreement is found between the theory and the FE results, both in terms of the absolute attenuation values and for asymptotic power relationships. In the geometric regime, power relationships are obtained through a combination of dimensional analysis and finite element simulations. The results here also provide useful insight in verifying the three-dimensional theory, since the method used for its derivation is analogous.

b) g.sarris18@imperial.ac.uk

c) Also at: Department of Mechanical Engineering, Imperial College London, London, SW7 1AY, United Kingdom 


\section{INTRODUCTION}

Elastic waves guided on the surface of a solid are well known to attenuate if the surface is not perfectly flat, due to scattering ${ }^{1}$. The attenuation of Rayleigh waves from rough surfaces has been described analytically but experimental validations have proved difficult to achieve over a wide range of parameters of the roughness. One reason is that mathematical models are valid in regions that are difficult to replicate in practice since they either require very low roughness, or predict high attenuation values, which would render the waves undetectable.

Early work to describe the attenuation of Rayleigh waves resulted in the derivation of expressions for waves on flat surfaces of solids whose material properties induced attenuation. For instance, Maris $^{2}$ derived expressions for the attenuation of Rayleigh waves on a dielectric crystal with arbitrary crystallographic orientation, where temperature and viscosity were also considered. Their work followed the experimental results of Salzmann et $a l .{ }^{3}$ who used lasers to measure the effect of the temperature and the frequency on the attenuation of Rayleigh waves propagating along quartz crystals. These early studies considered specific attenuation cases related to material properties, but did not take into account the roughness that unavoidably exists on all surfaces and which causes attenuation even when the waves propagate on perfect lossless elastic materials; the attenuation occurs by partial scattering of the waves from the geometric features of the roughness ${ }^{1}$.

Some of the first analytical expressions for the attenuation of Rayleigh waves from rough generalised surfaces were derived by Maradudin \& Mills ${ }^{4}$, and Urazakov \& Fal'kovskii ${ }^{5}$.

The rough surface was described using two statistical parameters - the root mean squared 
(RMS) height, $\delta$, which describes the amplitude of the peaks and troughs of the roughness, and the correlation length, $\Lambda$, which is a measure of the spacing of those peaks. Urazakov \& Fal'kovskii used the Rayleigh method for their studies. This method solves the Rayleigh wave equation, which predicts the creation of Rayleigh waves on a stress free boundary ${ }^{6}$, but was extended by the authors to apply that stress free surface condition across the rough surface.

Maradudin \& Mills used a Green's function approach to solve the relevant equations. Both approaches predict that for the three-dimensional case, when the roughness is low (for instance, $\delta / \Lambda<0.3)$ the attenuation coefficient is proportional to the fifth power of the wave's frequency, $f$, in the region where the Rayleigh wavelength, $\lambda_{R}$, is much greater than $\Lambda$. Maradudin \& Mills also demonstrated that the attenuation coefficient is proportional to $\delta^{2}$. Subsequently, Eguiluz \& Maradudin ${ }^{7}$ published an updated version of their derivations in which the additional scattering of the Rayleigh waves to bulk waves was considered. The new results also demonstrated a proportionality of the attenuation coefficient to $f^{5}$, as well as a new result of a proportionality to the ratio $\delta^{2} / \Lambda^{2}$.

Following the work by Eguiluz \& Maradudin, de Billy et al., ${ }^{8}$ completed some experimental work to verify the theory derived in ${ }^{7}$. In their work, attenuation measurements were taken for rough duraluminium and titanium samples, and the effect of varying $\lambda_{R}$ on the attenuation measurements was investigated. The authors found good agreement between their experimental results and the $f^{5}$ relationship predicted by the theory at the long wavelength limit, however, they observed that this $5^{\text {th }}$ order proportionality did not hold true at smaller $\lambda_{R}$ values. More recently, Kosachev \& Gandurin ${ }^{9}$, studied the dispersion atten- 
uation of Rayleigh waves on statistically rough hexagonal crystals, to expand the work in ${ }^{7}$. In their work, the scattering from the rough surface of an anisotropic hexagonal crystal was theoretically studied, with the authors deriving an expression for the attenuation coefficient when the scattering occurs at a generalised crystal orientation. Their expression reduces to the same $f^{5}$ relationship derived $i n^{7}$, for the isotropic case in the long wavelength region. Finally, Chukov ${ }^{10}$ used the Rayleigh-Born approximation to derive similar relationships to ${ }^{7}$ for the isotropic case, arriving at the same power relationships. In addition, Chukov derived expressions for 2D roughness which are discussed in more detail below.

However, the theory is restricted to specific $\delta / \Lambda$ and $\lambda_{R}$ combinations. In particular, a large proportion of the theory has been derived in the Rayleigh regime - this is a region where $\lambda_{R} \gg \Lambda$, which is a very low frequency regime. Therefore, the above-mentioned considerations motivate two research problems which this paper attempts to solve - firstly, to create a finite element (FE) model to validate the existing analytical expressions in the Rayleigh region, and secondly, to extend the results, by FE modelling, to other more practically relevant regimes.

To obtain a representative attenuation value for a combination of $\delta, \Lambda$ and $f$, from numerical modelling, it is necessary to average over a sufficiently large number of attenuation values obtained from individual surfaces characterised by those specific statistical parameters. It is also necessary to have a model sufficiently large to accommodate a representative scattering distance. The implementation of a 3D model would incur significant computational burden and run-times for such a wide range of parameter values and so this paper 
conducts a comprehensive $2 \mathrm{D}$ analysis. The validation in $2 \mathrm{D}$ also infers validation in $3 \mathrm{D}$, as the same theoretical approach has been implemented for the derivations in both cases.

In addition to the 3D case discussed above, Chukov demonstrated that for 2D the attenuation coefficient is proportional to $f^{4}, \delta^{2}$ and $\Lambda$ in the Rayleigh region in comparison with the $f^{5} \delta^{2} \Lambda^{2}$ which has been derived for 3D roughness. Identical power relationships were also derived by Huang \& Maradudin ${ }^{11}$, using the same small perturbation method implemented in the 3D analysis ${ }^{7}$, and the $f^{4}$ relationship was observed experimentally in ${ }^{8}$. These are the proportionality relationships which this paper validates. In addition to this, Maradudin \& Eguiluz ${ }^{7}$ and Huang \& Maradudin ${ }^{11}$ have also derived an expression which gives quantitative values for the attenuation coefficient - the results from the FE simulations in this study are also compared against this expression.

Following this validation, this paper looks into the attenuation coefficient's behaviour in regimes outside the Rayleigh region. More specifically, both the stochastic $\left(\lambda_{R}<\Lambda\right)$ and geometric $\left(\lambda_{R} \ll \Lambda\right)$ regimes are investigated. It is worth noting that in the literature, these scattering regimes are studied separately, or under different assumptions. In our study, the same approach was used across all three scattering regimes, creating a unified method for studying the scattering of Rayleigh waves from rough surfaces, regardless of the scattering regime.

Analytical expressions for the attenuation of Rayleigh waves from statistically rough surfaces are, to our knowledge, very limited in both the geometric and stochastic regions. However, a useful analogous problem has been studied in detail by Van Pamel et $a l .{ }^{12}$. In their study, the authors investigated wave scattering within heterogeneous media - where the 
wave's propagation was impeded by the presence of grain boundaries within a material. In the Rayleigh regime, the authors found a reduction of the attenuation coefficient dependence by one power of frequency between the $2 \mathrm{D}$ and $3 \mathrm{D}$ scattering - this is consistent with the reduction of the proportionality from $f^{5}$ to $f^{4}$ in $2 \mathrm{D}$, for roughness scattering, derived by ${ }^{10}$ $a^{11}$. The same fourth power proportionality between the attenuation coefficient and the frequency has also been derived by Kaganova \& Maradudin for the scattering of surface waves in a polycrystalline material ${ }^{13}$. Additionally, it was found that at large $\lambda_{R}$ values, belonging to the stochastic region, the attenuation coefficient is proportional to the same powers of $\delta, \Lambda$ and $f$, regardless of the number of dimensions. Therefore, for the problem studied here, the same power relationships derived for the 3D case, by Kosachev et al. ${ }^{14}$ can be suggested to hold true in 2D. Regarding the geometric region, the authors in $^{12}$ and $^{15}$ state that the attenuation coefficient is independent of $f$.

This paper is split into the following sections. The theory and analytical results are discussed in more detail in Section II. The process of setting up the FE model is described in Section III. The results from the FE simulations are presented and discussed in Section IV, and finally, Section V concludes the work.

\section{THEORY}

This section presents the theory related both to the generation of rough surfaces, and to the power relationships between the attenuation coefficient and the different parameters which characterise the incident wave and the rough surface. The analytical expressions for 
calculating the attenuation coefficient, $\alpha$, along with a brief derivation are also presented and discussed.

\section{A. Rough Surfaces}

The weighted moving average method described in ${ }^{1}$ and $^{16}$ was implemented to generate the rough surfaces. An important parameter that characterises a rough surface is its RMS height, $\delta$. This is a measure of the height of the surface's peaks and troughs, relative to a reference surface, whose RMS height is zero. Let $x$ be the direction in which the rough surface lies, and $z$ the direction perpendicular to $x$. Now, let $h$ be the distance between the $z=0$ line and a point on the rough surface. Using these definitions, the rough surface's height profile can be described by

$$
z=h(x) .
$$

For the rough surfaces used in this study, the mean height of the rough surfaces was set to be 0 , i.e. $\langle h\rangle=0$, where the angled brackets denote the ensemble average value of the quantity. Under this assumption, $\delta$ is given by:

$$
\delta=\sqrt{<h^{2}>} .
$$

A second parameter typically used when describing a rough surface is the correlation length, $\Lambda$. This can be considered a measure of the spacing between the peaks and troughs of the surface, in the $x$ direction. Mathematically, it is defined as the distance over which the correlation function, $C(R)$ drops to $1 /$ e from its initial value, for two points separated 
by a distance $R$, where the correlation function is defined as follows:

$$
C(R)=\frac{<h(x) h(x+R)>}{\delta^{2}} .
$$

In this study, a Gaussian $C(R)$ was chosen:

$$
C(R)=\exp \left(-\frac{R^{2}}{\Lambda^{2}}\right) .
$$

Gaussian roughness was selected for this study, as it has been widely studied and is also well understood ${ }^{1,16,17}$. Additionally, it has been shown that Gaussian roughness can occur naturally, as reported $i^{18}$ and $^{19}$, where real fatigue cracks and real surfaces were found to follow a Gaussian roughness profile. Therefore our choice does not restrict the analysis to an idealised domain.

To create the rough surfaces, initially a set of random numbers was generated. Then, equations (2) and (4) and the moving average approach described in $^{16}$ were implemented, to transform this set of random numbers to a set of correlated numbers, corresponding to the values in the $h(x)$ function.

\section{B. Power Relationships}

The usual dispersion relationship for a Rayleigh wave travelling on a flat surface is

$$
\omega=C_{R} q,
$$

where $\omega$ is the Rayleigh wave's angular frequency, $C_{R}$ is the propagation velocity and $q$ is the wavenumber. Although in most modern work, the wavenumber is denoted by $k$, the use of $q$ here is in alignment with Maradudin's notation, and facilitates the comparison between our 
results and previous work. When the Rayleigh wave encounters a rough surface, equation

(5) becomes $^{7}$

$$
\omega=C_{R} q+\Delta \omega,
$$

where $\Delta \omega$ is a complex angular frequency perturbation, arising from the presence of the rough surface.

Eguiluz \& Maradudin ${ }^{7}$ have shown that the attenuation length $l$, which is the length over which the Rayleigh wave's energy falls to $1 /$ e from its initial value, can be calculated via

$$
l^{-1}=2\left(\frac{\delta^{2}}{\Lambda^{2}}\right) q \omega_{2}
$$

where $\omega_{2}$ is a function encapsulating the effect of the roughness on the Rayleigh wave. It can be then shown that for $\lambda_{R} \gg \Lambda$ (Rayleigh region) $\omega_{2} \propto(\omega \Lambda)^{4}$, for 3D roughness, and hence, $l_{3 \mathrm{D}_{R}}^{-1} \propto f^{5} \delta^{2} \Lambda^{2}$, where the subscripts $3 \mathrm{D}$ and $R$ denote the presence of three-dimensional roughness, and the Rayleigh region respectively.

An analogous analysis in ${ }^{11}$ and $^{20}$ showed that for 2D roughness, the $\omega_{2}$ function is proportional to the third power of $\omega$ and $\Lambda$, in the Rayleigh regime. The derivations were performed using similar methods to those in ${ }^{7}$, which allows $\omega_{2}$ to be directly substituted into the right-hand side of equation (7). Therefore, the theory predicts that $l_{2 \mathrm{D}_{R}}^{-1} \propto f^{4} \delta^{2} \Lambda$, where the subscript $2 \mathrm{D}$ denotes the case of two-dimensional roughness.

For the stochastic region, in an inhomogeneous medium in $3 \mathrm{D}$, where $\lambda_{R}<\Lambda$, it has been shown analytically in ${ }^{12}$ that there is no difference in the power relationships in $3 \mathrm{D}$ and $2 \mathrm{D}$, between $l^{-1}$ and the relevant parameters - this observation was subsequently verified numerically by the authors. In this $\mathrm{study}^{12}$, the authors derived this theory for the attenuation arising from inhomogeneous media - however, their analysis used similar metrics to 
ours. The size of the "obstacle" causing the attenuation was characterised by its correlation length, and the stochastic regime was defined as the region where $q \Lambda>1$. We can therefore suggest the independence of the attenuation length, with respect to dimensionality, to hold true in our case as well, i.e. the same power relationship exists between $l^{-1}$ and $\delta, \Lambda$ and $f$ in both 3D and 2D roughness. Kosachev et al. ${ }^{14}$ have derived an analytical expression for the stochastic region for 3-dimensional roughness - therefore based on their derivation, we expect that $l_{2 \mathrm{D}_{S}}^{-1} \propto f^{2} \delta^{2} \Lambda^{-1}$, where the subscript $S$ denotes the stochastic region.

In order to present power relationships relating to the geometric regime, it is first necessary to introduce the dimensional analysis associated with the asymptotic study of the attenuation coefficient. The asymptotic study is based on the principle of similitude, which stipulates that the study of different phenomena can be treated using equivalent equations, if they can be described by the same dimensionless variables ${ }^{6}$. For studying attenuation phenomena, an equivalent mathematical analysis can be implemented if roughness parameters $(\delta$ and $\Lambda$ ) and the loss in energy $(\alpha)$ are normalised by the Rayleigh wavelength.

More specifically, the asymptotic approximations usually take the form of products of powers for the dimensionless normalised attenuation coefficient, $\alpha_{n}$ :

$$
\alpha_{n} \propto \delta_{n}^{m_{\delta}} \Lambda_{n}^{m_{\Lambda}},
$$

where $\alpha_{n}=\alpha \lambda_{R}, \delta_{n}=\delta / \lambda_{R}$ (normalised RMS height), $\Lambda_{n}=\Lambda / \lambda_{R}$ (normalised correlation length) and $m_{\delta}$ and $m_{\Lambda}$ are the powers of $\delta_{n}$ and $\Lambda_{n}$ to which $\alpha_{n}$ is proportional. Given that unperturbed Rayleigh waves travelling on a smooth flat surface are non-dispersive, the frequency is inversely proportional to the wavelength, and therefore equation (8) can be 
rewritten as:

$$
\alpha_{n} \propto \delta^{m_{\delta}} \Lambda^{m_{\Lambda}} f^{m_{\delta}+m_{\Lambda}},
$$

or equivalently,

$$
\alpha \propto \delta^{m_{\delta}} \Lambda^{m_{\Lambda}} f^{m_{f}},
$$

where

$$
m_{f}=m_{\delta}+m_{\Lambda}+1 \text {. }
$$

Equation (11) holds true regardless of the number of dimensions and the scattering regime - this can also be confirmed by observing both the $3 \mathrm{D}$ and $2 \mathrm{D}$ power relationships demonstrated in the previous paragraphs, which all obey equation (11). In the geometric regime, the scattering is independent of the frequency ${ }^{15}$. Therefore, from equation (11), $m_{\delta_{G}}=-1-m_{\Lambda_{G}}$, and $l_{2 D_{G}}^{-1} \propto \delta^{m_{\delta_{G}}} \Lambda^{-1-m_{\delta_{G}}}$, where the subscript $G$ denotes the geometric regime. Here, the power coefficients relating to $\delta$ and $\Lambda$ will be treated as unknowns to be found, and the independence of the attenuation coefficient on $f$ will be validated by the FE model.Finally, it is worth noting that for all the theoretical derivations, it was assumed that $\delta<\Lambda$, which follows numerous studies on rough surface scattering in the literature, such $\operatorname{as}^{18,21-23}$.

In our analysis, we will be using a similar attenuation measure to $l^{-1}$ : the attenuation coefficient $\alpha$, which is defined as the inverse of the distance over which the amplitude of the Rayleigh wave drops to 1 /e from its initial value. Using this definition, in combination with the fact that the energy of a wave is proportional to the square of its amplitude ${ }^{24}$, we can 
TABLE I. Expected asymptotic power relationships, between the attenuation coefficient and the RMS height, correlation length and frequency. In this table, $q$ is the wavenumber, $\delta$ is the RMS height, $\Lambda$ is the correlation length and $f$ is the frequency.

\begin{tabular}{|c|c|c|c|}
\hline Regime & Rayleigh & Stochastic & Geometric \\
\hline Limits & $q \delta<q \Lambda<1$ & $q \delta<1<q \Lambda$ & $1<q \delta \ll q \Lambda$ \\
\hline$\alpha(\delta, \Lambda, f)$ & $\delta^{2} \Lambda f^{4}$ & $\delta^{2} \Lambda^{-1} f^{2}$ & $\delta^{m_{\delta_{G}}} \Lambda^{-1-m_{\delta_{G}}}$ \\
\hline$\beta\left(\delta_{n}, \Lambda_{n}\right)$ & $\delta_{n} \Lambda_{n}^{2}$ & $\delta_{n}$ & $\delta_{n}^{m_{\delta_{G}}-1} \Lambda_{n}^{-m_{\delta_{G}}}$ \\
\hline
\end{tabular}

relate $\alpha$ to $l$ using the following equation:

$$
\alpha=\frac{1}{2 l}
$$

A summary of the expected power relationships between $\alpha$ and $\delta, \Lambda$ and $f$ is shown in Table I. For ease and uniformity of presentation, we have introduced the dimensionless notation $\delta_{n}, \Lambda_{n}, \alpha_{n}$ and $\beta$, where $\alpha_{n}=\alpha \lambda_{R}$ and $\beta=\alpha_{n} \Lambda / \delta$. The variable $\beta$ is defined to later allow us to generate a master curve where we plot the numerical results against a single variable. If just the conventional normalised attenuation coefficient $\left(\alpha_{n}\right)$ is used, this yields results which are functions of both $\delta_{n}$ and $\Lambda_{n}$, making it impossible to plot all the results in a single graph, as they are not functions of a single variable. The variable $\beta$ is defined such that all the numerical results become a function of a sole variable $\left(\delta_{n}\right)$, assuming that $m_{\delta_{G}}$ is zero. 


\section{SETTING UP THE FINITE ELEMENT MODEL}

This section presents the method used to create the FE models, which were used in all subsequent simulations. The purpose of the FE models is to allow us to study the phenomenon of surface wave scattering from a large range of roughness parameters, meaningfully and efficiently. Therefore, the necessary steps were taken to minimise the model's size and to ensure that the surface wave was of good quality, with minimal noise. The process to achieve these properties, as well as the method used to calculate the attenuation coefficient, and the computational resources used, are presented below.

Despite the rough surfaces being characterised by their RMS height and correlation length, each surface has a unique $h(x)$ profile. In order to obtain a meaningful value for attenuation, it was necessary to perform Monte Carlo simulations, and average over a sufficient number of realisations for each $\delta$ and $\Lambda$, to ensure the statistical stability of the result. The simulations were conducted using the high-fidelity, GPU-based FE software package Pogo, which is an explicit time domain finite element solver and visualised using PogoPro $^{25}$.Following the discretisation of the domain, Pogo uses the well-known finite difference method, with the aid of a stress-free boundary condition, to obtain the displacement at each node. If we denote the displacement, velocity, and acceleration matrices of the nodes in the model by $\mathbf{U}, \dot{\mathbf{U}}$ and $\ddot{\mathbf{U}}$, the equation for elasticity theory becomes:

$$
\mathbf{M U ̈}+\mathbf{C} \dot{\mathbf{U}}+\mathbf{K U}=\mathbf{F},
$$

where $\mathbf{M}, \mathbf{C}$ and $\mathbf{K}$ are the mass, damping and stiffness matrices, and $\mathbf{F}$ is the applied force matrix. By implementing the finite difference method, and assuming a model with no 
damping terms, Equation (13) becomes:

$$
\mathbf{M} \frac{\mathbf{U}^{n+1}-2 \mathbf{U}^{n}+\mathbf{U}^{n-1}}{\Delta t^{2}}+\mathbf{K} \mathbf{U}^{n}=\mathbf{F},
$$

where the superscript $n$ denotes the corresponding matrix at the $n^{\text {th }}$ time step, and $\Delta t$ is the duration of that time step. By rearranging Equation (14), the displacement at the $n+1$ time step can be found, using the values for the displacement at the previous two time steps. The approach described here follows closely the approach taken by other high-fidelity FE studies for elastic wave propagation ${ }^{26-28}$.

Inconel 718 was used in all simulations (Young's modulus, $E=208.73 \mathrm{GPa}$, Poisson's ratio, $\nu=0.303$ and density, $\left.\rho=7800 \mathrm{~kg} / \mathrm{m}^{3}\right)$. For a given material, the Rayleigh wavespeed $C_{R}$, can be calculated approximately by the following formula ${ }^{29}$ :

$$
C_{R}=\frac{0.862+1.14 \nu}{1+\nu} C_{S},
$$

where $C_{S}$ is the shear wave speed. For the material parameters defined above, $C_{R}$ was found to be $2892 \mathrm{~m} / \mathrm{s}$.

Each rough surface was inserted to form the lower boundary of a 2D rectangular FE domain with the specified material parameters. The length of the rough surface was set to be at least $50 \Lambda$, to ensure statistical and ergodic stability ${ }^{1}$. A Tukey window was applied the rough surface, to ensure a smooth joining with the main material and avoid generating additional artificial attenuation. The Tukey window function, $w(x)$, was of the following 


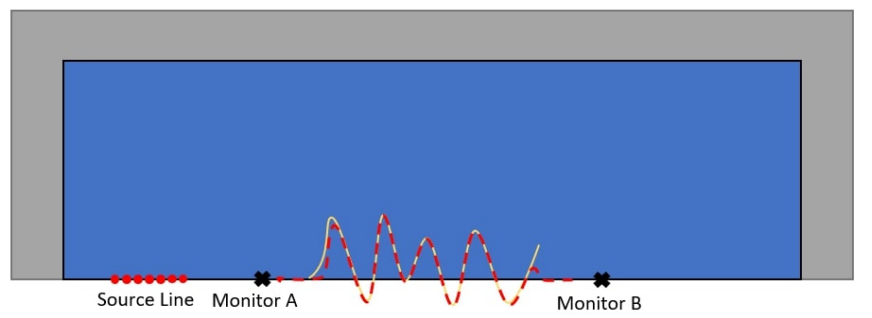

where $L$ is the length of the rough surface, and $l_{w}$ is the length of the window tapering. For our simulations, $l_{w}=\frac{L}{10}$ was used. The windowing was implemented by multiplying $w(x)$ with $h(x)$ - this has the effect of smoothing the edges of the surface, while leaving the rest of it unaffected. A schematic of the FE model is shown in Figure 1. Two-dimensional Absorbing layers

* Monitor nodes

- Source nodes

- Original rough surface

(prior to insertion)

Rough surface used in the FE model (Tukey window applied)

FIG. 1. (Colour online) Schematic of the FE model. A Tukey window is applied to the original rough surface, generated using the method described in subsection II A (in yellow), before it is inserted to form the lower boundary of the FE domain (in red). The scale of the rough surface is exaggerated for better visualisation.

triangular elements were used in generating the mesh. The mesh size, $\Delta x$, was set to be approximately equal to $\lambda_{R} / 25$, where $\lambda_{R}$ was calculated from the central frequency of each simulation, for all models - this is necessary to avoid errors in the elastic wave speed, and ensure the numerical stability of the model, which are issues that might arise if the mesh is 
too coarse, as described in ${ }^{30}$. A similar approach for the mesh size has been used in studies related to ours ${ }^{31}$. A typical model size was of the order of $2 \times 10^{6}$ degrees of freedom.

An example of the lower portion of the FE domain, after the rough surface was attached to its lower boundary, is shown in Figure 2. There are two interesting features in Figure 2, firstly, the ability of Pogo to mesh efficiently can be observed, as the irregular mesh only lies close to where the rough surface is located, while the mesh efficiently reverts to a regular form in the main bulk of the material as the distance from the rough surface increases. Secondly, the smooth joining of the rough surface to the FE domain can also be seen. Here, it is worth acknowledging that rough surfaces can be described by fractals ${ }^{32}$, with past roughness studies using the Weierstrass function ${ }^{33}$, which exhibits self-similarity, to model them. Meshing unavoidably truncates this fractal feature. However, it is expected that the absence of this fractal nature will not affect the result, as geometrical features significantly smaller than the wavelength can not be resolved by the wave $\mathrm{e}^{22,34}$.

In the FE model, the input signal used was a 5-cycle Hann windowed tone burst. The absolute value of signal's amplitude was arbitrarily selected, since the simulation is linear, and we are concerned about ratios of measured results, and not their absolute values. A source line, comprised of multiple source nodes, was located to the left of the rough surface. For each simulation, the size of the source was set to be equal to three Rayleigh wavelengths, calculated from the simulation's central frequency. To obtain a Rayleigh wave travelling towards the rough surface, a phase delay was applied to each node, such that constructive interference from the signal from each node occurred in the desired direction. This method was implemented as follows: 


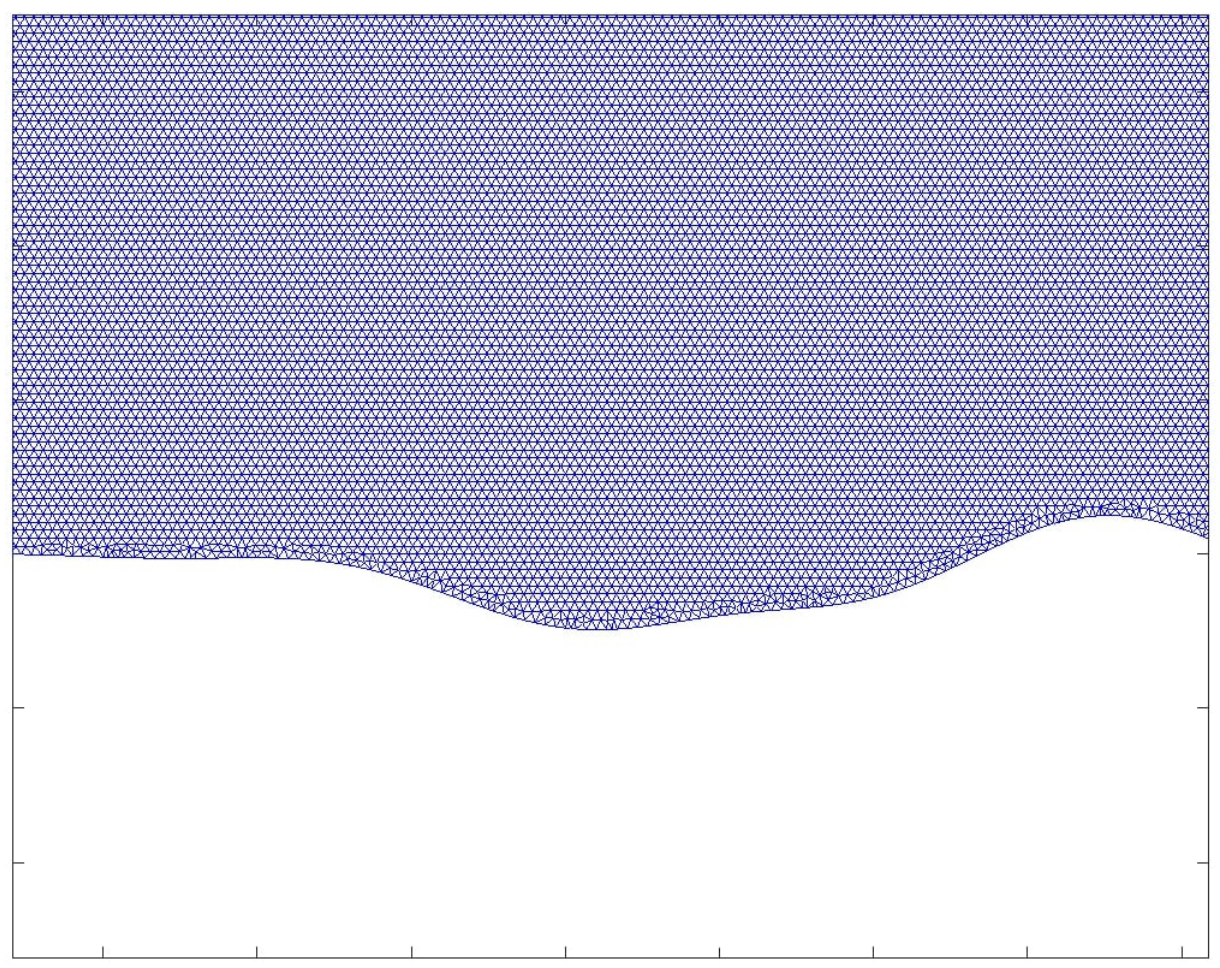

FIG. 2. (Colour online) Detail of the FE domain's meshing, after the Tukey-windowed rough surface has been applied to its lower boundary.

- Two sinusoidal time-domain signals were created, with a $90^{\circ}$ phase shift between them.

- Pogo provides the ability to assign each source node a unique amplitude, which scales the time domain signal assigned to that node accordingly. Therefore, the amplitude at each node was selected in a way such that a clean Rayleigh wave, with the correct amplitude and phase was created by the interference of the signal from all the nodes.

- The complex amplitude assigned the $i^{\text {th }}$ source node, $a_{i}$, located at the $x_{i}$ position in the model is given by:

$$
a_{i}=\frac{1}{2}\left[1-\cos \left(2 \pi \frac{x_{i}-x_{\min }}{x_{\max }-x_{\min }}\right)\right] e^{j q x_{i}}
$$


where, $x_{\min }$ is the position of the leftmost source node, $x_{\max }$ is the position of the rightmost source node and $j$ is the imaginary unit. The collective use of a unique amplitude at each node, according to equation (17), results in the constructive interference of the signals from all source nodes, in the correct direction, which generates a clean Rayleigh wave.

- The first signal was applied to the $i^{\text {th }}$ node with a weighting of $\left[\operatorname{Im}\left(a_{i}\right),-\operatorname{Re}\left(a_{i}\right)\right]$ and the second signal with a weighting of $\left[-\operatorname{Re}\left(a_{i}\right),-\operatorname{Im}\left(a_{i}\right)\right]$, where the two entries in the previous vectors denote the $x$ and $z$ direction respectively, and the notations $\operatorname{Re}()$ and $\operatorname{Im}()$ denote the real and imaginary part of their argument respectively. The amplitudes of the $x$ and $z$ components of the Rayleigh wave are arbitrary since one is interested in the ratio of the amplitudes before and after the rough surface, rather than the absolute values.

An example of a Rayleigh wave created using the method described above is shown in Figure 3. As shown in the figure, a pure Rayleigh wave is created by implementing this method. The minimal secondary waves which exist in Figure 3, just above the right-hand end of the Rayleigh wave, lie away from the rough surface and therefore do not interfere with the attenuation measurements.

In order to obtain the information required to calculate the attenuation coefficient, two monitor nodes were used in the model, one on either side of the rough surface. The Rayleigh wave's z-amplitude was measured at each monitor node. The attenuation coefficient was then 
FIG. 3. (Colour online) Example of a Rayleigh wave field, travelling in the positive $x$-direction created using the method described in this section. The colour scale in the figure represents the absolute magnitude of the displacement at each node. The Rayleigh wave's centre frequency is $6 \mathrm{MHz}$ and the rough surface has $\delta=25 \mu \mathrm{m}$ and $\Lambda=50 \mu \mathrm{m}$.

calculated by our definition of $\alpha$ :

$$
\alpha=-\frac{1}{x_{d}} \ln \left(\frac{A_{2}}{A_{1}}\right),
$$

where $A_{1}$ and $A_{2}$ are the amplitudes of the Fourier transforms of the Rayleigh wave before and after the rough surface, and $x_{d}$ is the distance between the locations at which $A_{1}$ and $A_{2}$ were obtained.

Finally, in order to avoid unwanted noise from the source, or scattered waves interfering with the attenuation measurements, absorbing layer regions were applied to the top, left and right sides of the FE domain. These regions are defined by material parameters whose damping gradually increases, leading to attenuation rather than reflection of the waves ${ }^{35}$. 
The addition of absorbing layers in similar setups has proven to be beneficial in related rough surface studies ${ }^{36}$.

For each Monte Carlo simulation, 100 unique surfaces, and hence FE domains, were generated. Using 1 Nvidia GTX 1080Ti with 11 GB of memory, each set of 100 models takes about 1.5 hours to complete. This efficiency allowed one to run several statistically stable sets of simulations, covering a wide range of roughness, which better validates the theoretical models. A 3D approach would not have been able to achieve such wide variety of simulation parameter values due to computational limitations. The $2 \mathrm{D}$ study presented here provides useful and meaningful insight in the verification of the existing $3 \mathrm{D}$ theory, as the $2 \mathrm{D}^{11}$ and $3 \mathrm{D}^{7}$ theory have been derived under the same assumptions and using similar methods. Additionally, the 2D work here identifies the most important regimes 3D investigations may be conducted. It is also worth noting that the same method described here was used to generate all the FE models required for our study, regardless of the scattering regime. This strengthens the universality of our findings, as it has eliminated the need to study each regime separately, which is often the case in the literature.

\section{RESULTS \& DISCUSSION}

This section presents the results from our FE simulations, and is split into three parts. In the first part of this section, our FE results are compared quantitatively with equation (7), in order to investigate the agreement between the theory and the FE model. After this agreement is established, the second part of this section presents the results relating to the power relationships presented in Table I. 
The initial roughness statistical parameters, used for the Rayleigh regime, were selected to reflect values of roughness which can be found on metal parts created by additive manufacturing. More specifically, values of $\delta$ in the range of $10 \mu \mathrm{m}-25 \mu \mathrm{m}^{37-39}$ were chosen. The correlation length values were then adopted such that they fulfilled the $\delta<\Lambda$ condition, as required by the limiting conditions in Table I. When the investigation relating to the Rayleigh regime was completed, we explored the stochastic and geometric regimes by expanding our initial selection of $\delta$ and $\Lambda$ values.

Each surface realisation, despite being characterised by its $\delta$ and $\Lambda$ values, has a unique profile, due to the inherent randomness of roughness. It is therefore necessary to average over a sufficient number of realisations to get results which are statistically meaningful. Each datapoint in all the figures in this section, is an ensemble average $\alpha$ value, obtained from the Monte Carlo simulation of 100 realisations. This number of realisations lies within the range of 50-200 which has been used in similar studies ${ }^{22,26,31}$, but has also been verified for its statistical stability for our specific study, by conducting a convergence analysis, similar to that $\mathrm{f}^{40}$. An example of the convergence plots, for three roughness cases, is shown in Figure 4.

As shown in Figure 4, the number of realisations required to obtain a converged $\alpha$ value is a function of the surface roughness. For the case with the longest correlation length in Figure 4 , the $\alpha$ value converges after approximately 15 realisations, while for the other two cases, where the correlation length is shorter, and therefore the peaks and troughs of the surface are closer together, a higher number of realisations is required for convergence. 


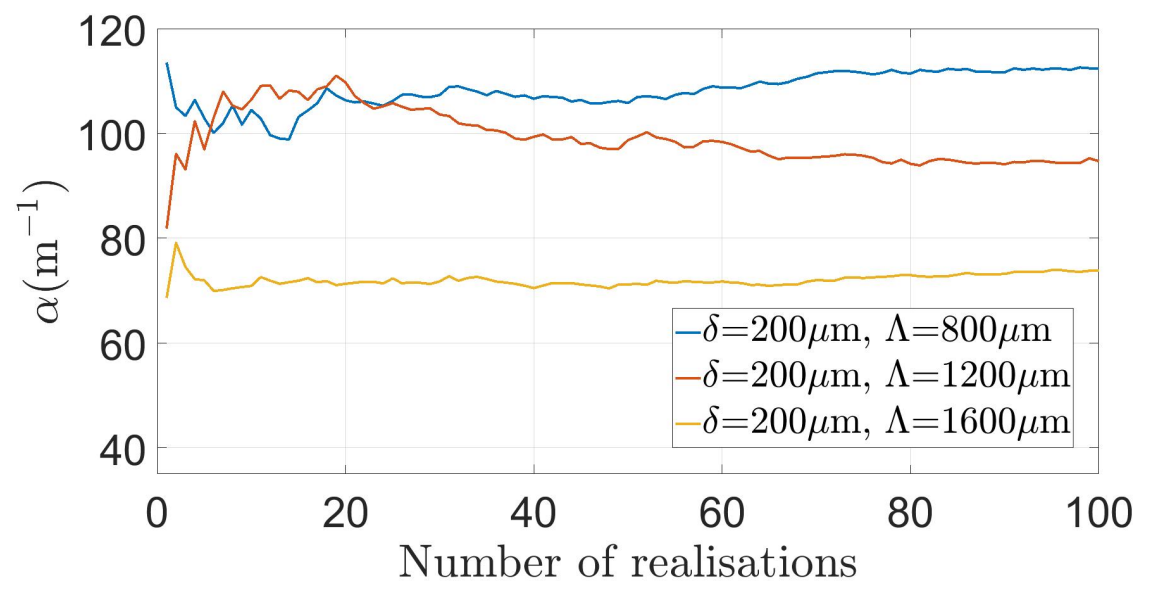

FIG. 4. (Colour online) Variation of the attenuation coefficient, as the number of realisations increases, for three roughness scenarios, at $f=10 \mathrm{MHz}$.

However all cases converged before the 100 realisation limit was reached, further supporting our choice.

Finally, following the verification of the quantitative and asymptotic results, the last part of this section presents a "master" attenuation curve, on which attenuation values from various $\delta, \Lambda$ and $f$ combinations are plotted, as a method to further verify the agreement of the FE and the theory, over a wider range of parameters. A summary of all the power relationships obtained by FE modelling is also given.

\section{A. Quantitative Results}

A comparison between the theoretical $\alpha$ and the values predicted by combining equations (7) and (12) is shown in Figure 5. The statistical parameters used to obtain this figure were $\delta=200 \mu \mathrm{m}, \Lambda=800 \mu \mathrm{m}$, and the frequency was varied from $0.4 \mathrm{MHz}$ to $1.75 \mathrm{MHz}$. 


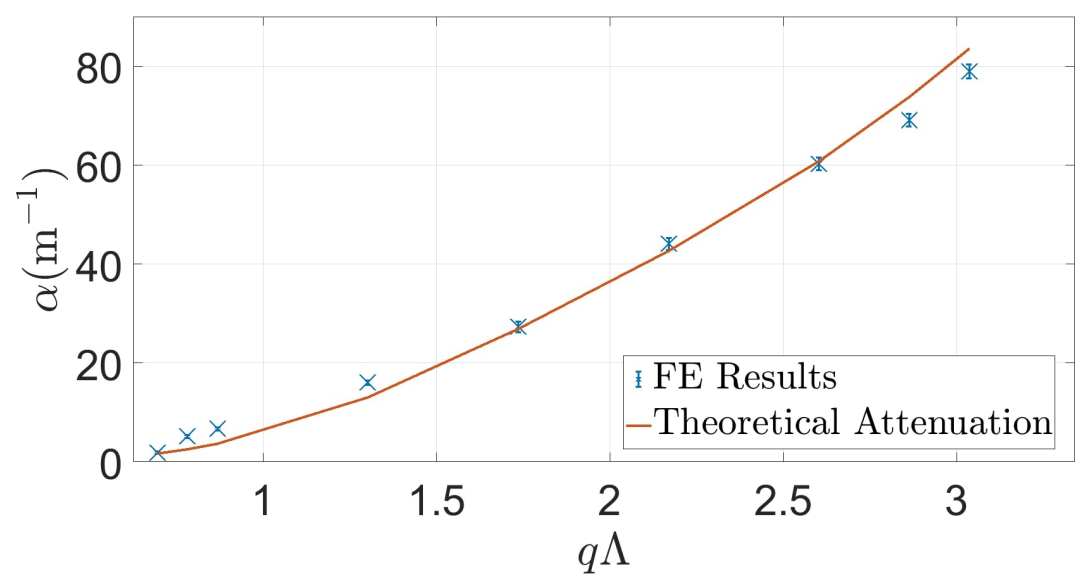

FIG. 5. (Colour online) Comparison of theoretical $\alpha$ values with FE results. The attenuation coefficient is plotted against the dimensionless quantity $q \Lambda$, which is analogous to plotting against frequency. The theoretical predictions of the model of ${ }^{11}$ are shown using the curve, and the FE results are plotted as $\times$.

In the horizontal axis of Figure 5, the dimensionless quantity $q \Lambda$ is plotted - this is analogous to plotting against frequency, as in all simulations $\Lambda$ was fixed, and the wavenumber $q$ varies linearly with frequency. Presenting the results as such also follows the work of Huang $\&$ Maradudin $^{11}$, who also plot against $q \Lambda$. The mean attenuation coefficient value is plotted as $\times$, and the plot includes error bars whose length is equal to \pm 2 standard errors (SE), where SE is defined as:

$$
\mathrm{SE}=\frac{\sigma}{\sqrt{n}} .
$$

In Equation (19), $\sigma$ is the standard deviation, and $n$ is the number of realisations.

It is clear that the FE results follow the curve predicted by the theory. The agreement is also evident in more than one of the scattering regimes described in subsection II B, since Figure 5 contains results for $q \Lambda$ values both greater and smaller than 1 , which is 
defined as the value at which the scattering behaviour changes from the Rayleigh to the stochastic regime. Using the definitions in Table I, the results of Figure 5 cover the Rayleigh and stochastic regions, with some of the points at higher $q \Lambda$ values lying in the transition region between the stochastic and geometric regimes. It is worth noting that there are some very small discrepancies between the theoretical attenuation coefficient and the FE results. However, we are looking at differences between two approaches here, so it would be inappropriate to view this as errors in the FE simulation not matching the theory. We believe there are three possible sources of discrepancies - approximations in the theory, errors in the FE simulations and insufficient convergence of the attenuation coefficient at 100 realisations. The outcome of recent studies using high-fidelity FE analysis is that the results from FE modelling are highly accurate. The authors in ${ }^{26-28}$ discuss in detail the high degree of accuracy achieved through FE, hence the error associated with the FE approach here is expected to be very small. Regarding the insufficient convergence issue, the results in Figure 4 show good convergence, hence we expect this error to also be small. Therefore, it is possible, indeed likely, that the approximations in the theory are a bigger contributor to the differences between the theory and the FE results. This confirms the validity of our FE model and allows us to proceed with a deeper analysis of each scattering regime.

\section{B. Power Relationships}

In this section, we present the results relating to the power relationships in Table I. In order to calculate the power relationship between $\alpha$ and the variable of interest, the sought power coefficient was determined numerically by a least squares regression analysis and the 
results are plotted on a log-log scale where the power coefficient of the best fitting power function is represented by the slope of the regression line. Similarly to Figure 5, standard error bars have been added to all the plots relating to the power relationships.

\section{Rayleigh Regime}

The results relating to the Rayleigh region are shown in Figure 6. We are plotting against the characteristic parameters $f, \delta_{n}$ and $\Lambda_{n}$ respectively, which are the frequency and normalised RMS height and correlation length respectively, as defined in subsection II B. According to the theory presented in subsection II B, we are expecting $\alpha \propto f^{4} \delta^{2} \Lambda$ in the Rayleigh regime. Figure 6(a) shows the simulation data, relating to the $f^{4}$ relationship. To generate the datapoints in Figure 6(a), the rough surfaces were defined to have $\delta=10 \mu \mathrm{m}$ and $\Lambda=20 \mu \mathrm{m}$. The frequency was varied from $1.75 \mathrm{MHz}$ to $2.25 \mathrm{MHz}$, and at each frequency point, a Monte Carlo simulation of 100 realisations was completed. The gradient of the best fit line in Figure 6(a) is 3.77, which is close to the expected value of 4.

Figure $6(\mathrm{~b})$ shows the simulation results, relating to the $\delta^{2}$ relationship. To produce Figure 6(b), the frequency of the simulations was set to $0.5 \mathrm{MHz}\left(\lambda_{R}=5800 \mu \mathrm{m}\right)$, and $\Lambda$ was set to $80 \mu \mathrm{m}$. Then, $\delta$ was varied from $30 \mu \mathrm{m}$ to $80 \mu \mathrm{m}$. The gradient of the best fit line in Figure 6 (b) is 1.77 , which is fairly close to 2 . It is worth noting that the power relationship here is calculated for $\alpha_{n}\left(\delta_{n}\right)$, however, it holds true against $\delta$ as well - this is because the simulations were completed at a fixed frequency (hence wavelength), and therefore the values in Figure 6(b) have been normalised by the same scalar. 


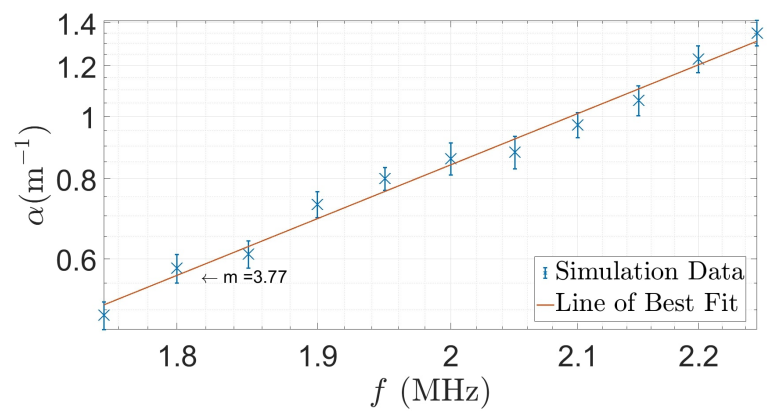

(a)

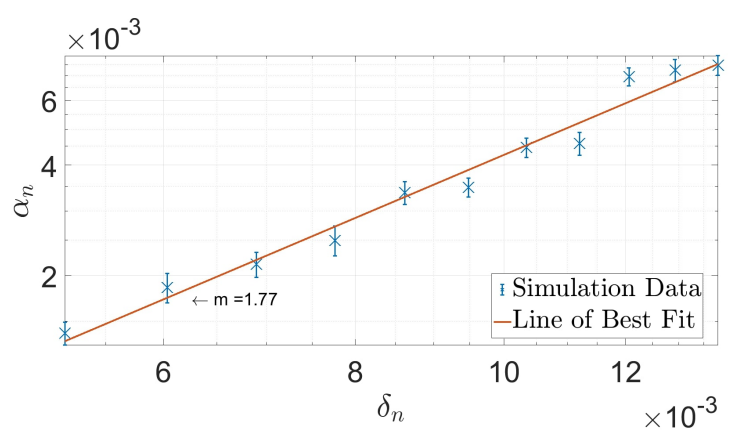

(b)

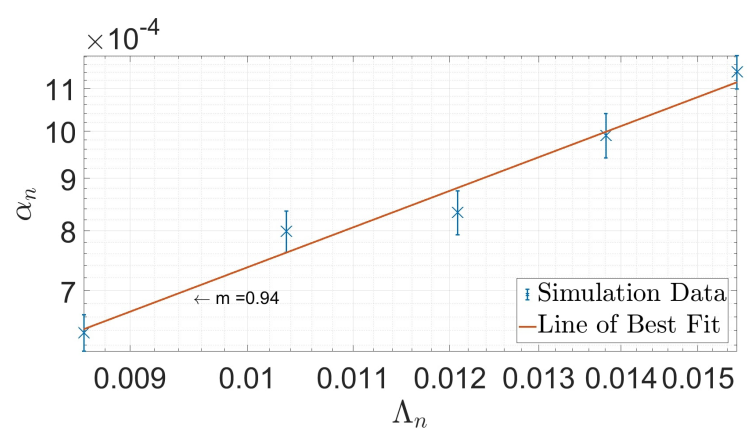

(c)

FIG. 6. (Colour online) FE results, relating to the Rayleigh regime. The figure shows the FE results, plotted as $\times$, and the line of best fit through them. The gradient of the best fit line, $\mathrm{m}$, is also shown on the figure. Values of the attenuation coefficient (either absolute or normalised) are plotted on the vertical axis, while the variable whose power relationship is investigated, is plotted on the horizontal axis. 
Figure 6(c) shows the simulation results relating to the $\Lambda$ relationship. To produce Figure $6(\mathrm{c})$, the frequency of the simulations was set to $0.5 \mathrm{MHz}\left(\lambda_{R}=5800 \mu \mathrm{m}\right)$ and $\delta$ was set to $20 \mu \mathrm{m}$. Then, $\Lambda$ was varied from $50 \mu \mathrm{m}$ to $90 \mu \mathrm{m}$. The gradient of the best fit line in Figure 6(c) is 0.94. Again even though the power relationship has been calculated for $\alpha_{n}\left(\Lambda_{n}\right)$, it remains the same for against $\Lambda$, for the same reason explained in the previous paragraph.

It appears that our FE results match the expected power relationships presented in Table I very closely. The power relationships can be explained as follows: The analysis in Maradudin \& Huang ${ }^{11}$ demonstrated that the $\omega_{2}$ function is proportional to $(\omega \Lambda)^{3}$ as $q \Lambda$ tends to 0 . When this is substituted into equation (7), it yields the $\alpha \propto f^{4} \delta^{2} \Lambda$ relationship, demonstrated by our FE results here. Physically, the attenuation coefficient tends to zero at low frequencies, because the Rayleigh wavelength becomes so long relative to the statistical parameters characterising the surface, that the surface appears flat to the wave.

\section{Stochastic Regime}

The results relating to the stochastic region are shown in Figure 7. Based on the theory presented in subsection II B, a proportionality of $\alpha$ to $f^{2} \delta^{2} \Lambda^{-1}$ is expected in the stochastic regime.

Figure 7(a) shows the FE results relating to the $f^{2}$ relationship. For this set of simulations, $\delta$ was set to $200 \mu \mathrm{m}$ and $\Lambda$ was set to $800 \mu \mathrm{m}$. The gradient of the best fit line is 1.94 . To produce Figure $7(\mathrm{~b})$, the frequency of all simulations was set to $1 \mathrm{MHz}\left(\lambda_{R}=2900 \mu \mathrm{m}\right)$, and $\Lambda$ to $800 \mu \mathrm{m}$. Then, $\delta$ was varied from $100 \mu \mathrm{m}$ to $400 \mu \mathrm{m}$, satisfying the stochastic region's condition. The gradient of the best fit line was found to be 2.15. Finally, Figure $7(\mathrm{c})$ was 


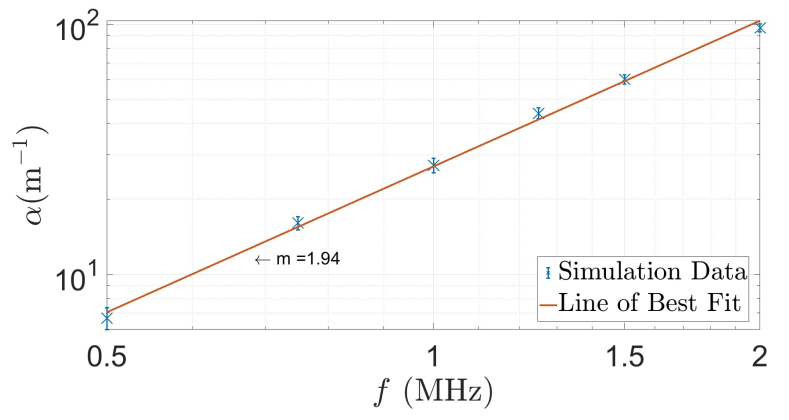

(a)

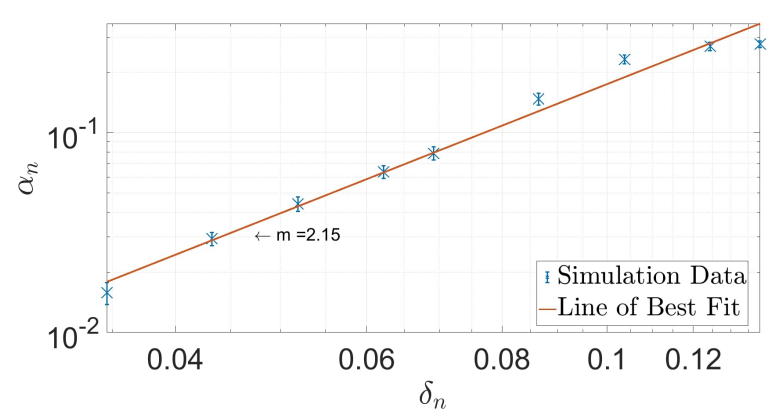

(b)

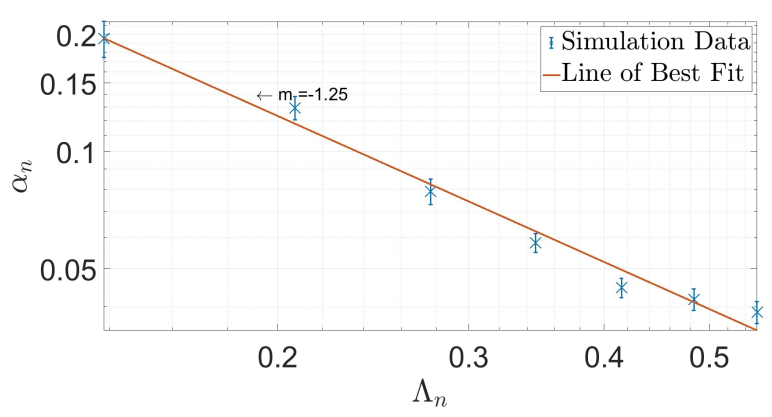

(c)

FIG. 7. (Colour online) FE results, relating to the stochastic regime. The figure shows the FE results, plotted as $\times$, and the line of best fit through them. The gradient of the best fit line, $\mathrm{m}$, is also shown on the figure. Values of the attenuation coefficient (either absolute or normalised) are plotted on the vertical axis, while the variable whose power relationship is investigated, is plotted on the horizontal axis. 
generated by setting the frequency again to $1 \mathrm{MHz}$. Then, $\delta$ was fixed to $200 \mu \mathrm{m}$, and $\Lambda$ was varied from $400 \mu \mathrm{m}$ to $1600 \mu \mathrm{m}$. The gradient of the best fit line was found to be -1.25 .

Overall, it appears that our FE model follows the already established theory in the stochastic region well. The power relationships here can be again explained by the behaviour of the $\omega_{2}$ function in the stochastic region. As has been demonstrated in ${ }^{11}$, in the stochastic region, $\omega_{2} \propto \omega \Lambda$. When this relationship is substituted in equation (7), it yields the $f^{2} \delta^{2} \Lambda^{-1}$ proportionality predicted by the theory and supported by our FE results.

\section{Geometric Regime}

The results relating to the geometric region are shown in Figure 8. The geometric region is a region where the RMS height is greater than $\frac{\lambda_{R}}{2 \pi}$, as per the definition made in Table I. Therefore, the frequency in this set of simulations was set to $5 \mathrm{MHz}\left(\lambda_{R}=580 \mu \mathrm{m}\right)$. Then, $\delta$ was set to $200 \mu \mathrm{m}$ and $\Lambda$ was varied from $800 \mu \mathrm{m}$ to $1600 \mu \mathrm{m}$. The gradient of the best fit line in Figure 8 is -0.83 .

The attenuation coefficient can be seen to be decreasing with an increase in frequency in Figure 8. Physically, in this region the wavelength has become so small compared with the correlation length, that the roughness does not impede its motion - the wave travels along the peaks and troughs without being scattered. Additionally, from the dimensional analysis presented in Section II B, the fact that $m_{\Lambda_{G}}$ is approximately equal to 1 implies that $m_{\delta_{G}}$ is approximately equal to zero in the geometric regime. This is further investigated and validated in the next subsection. 


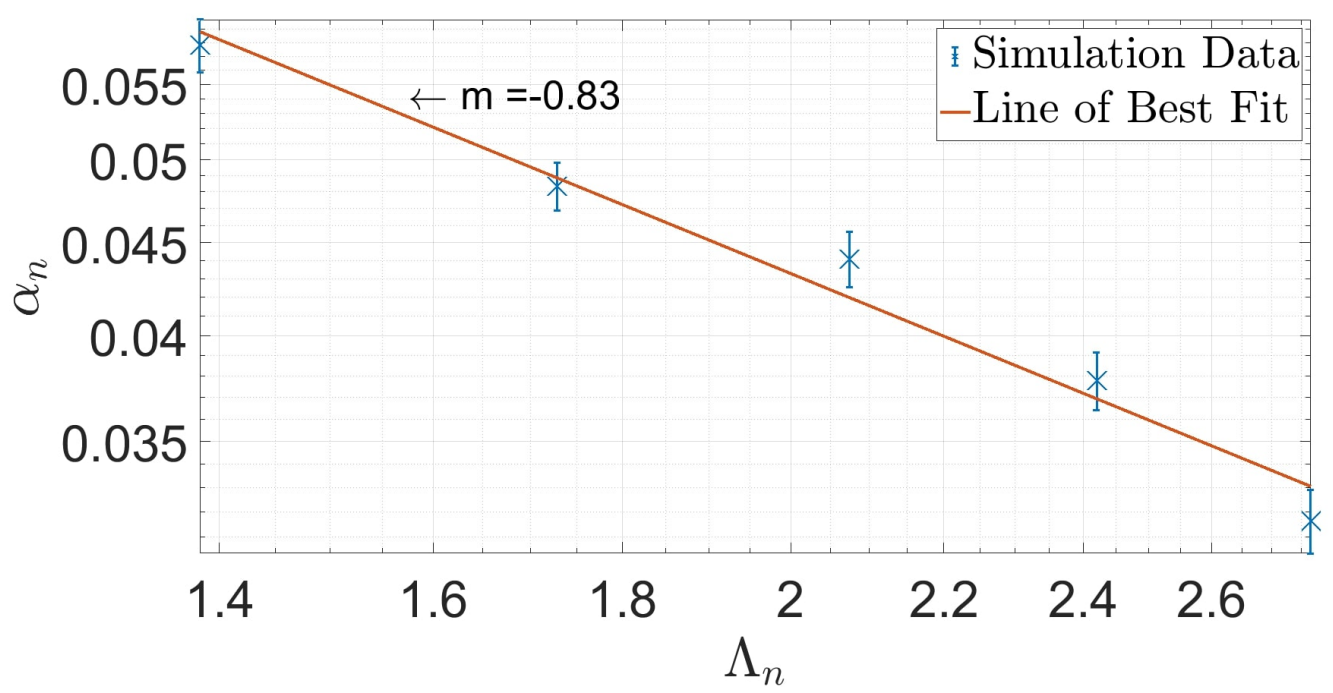

FIG. 8. (Colour online) $\alpha_{n}$ vs $\Lambda$ plot, geometric regime. The figure shows the FE results, plotted as $\times$, and the line of best fit through them. The gradient of the best fit line, $\mathrm{m}$, is also shown on the figure. Values of the attenuation coefficient (either absolute or normalised) are plotted on the vertical axis, while the variable whose power relationship is investigated, is plotted on the horizontal axis.

\section{Summary of results}

In Table I, we have also introduced the generalised attenuation coefficient, $\beta$. Using this allows us to further verify the validity of the theory in the stochastic and geometric regions, plotting a wider range of Monte Carlo results, against only the variable $\delta_{n}$. As shown in Figure 9, the results follow the asymptotic approximation lines, independently of which parameter was the variable in each FE Monte Carlo set. Additionally, the transition between the stochastic and the geometric region can clearly be seen at $\delta_{n}=1 / 2 \pi$. This is the expected location of the transition, and can be derived by identifying that in Table I, 


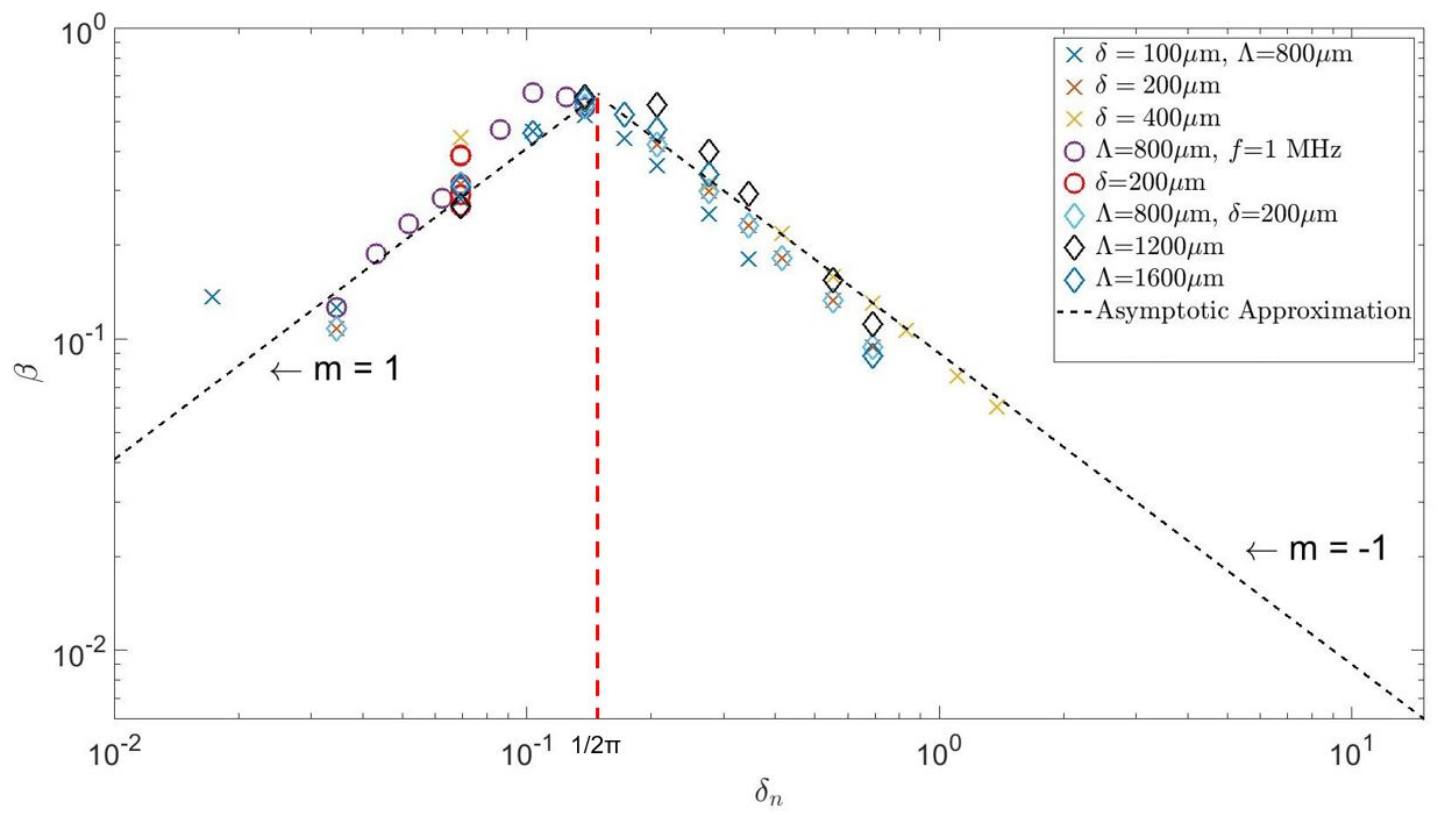

FIG. 9. (Colour online) $\beta$ vs $\delta_{n}$ master plot. Here, generalised attenuation coefficient values are drawn for a large combination of frequencies and roughness parameters. The datapoints cover both the stochastic and geometric regions, where the above proposed ad hoc approximation predicts that $\beta \propto \delta_{n}$ and $\beta \propto \delta_{n}^{-1}$ respectively, with the transition occurring at the $\delta_{n}=1 / 2 \pi$ point. The Monte Carlo results with a fixed $\Lambda(=800 \mu \mathrm{m})$ are plotted as $\times$, the results with a common $f(=1 \mathrm{MHz})$ are plotted as $\circ$, and the results with a fixed $\delta(=200 \mu \mathrm{m})$ are plotted as $\diamond$. The dashed black lines show the asymptotic approximation for both scattering regimes, while the red dashed line indicated the transition point between them.

the transition point between the stochastic and geometric regimes is defined to be where $q \delta=1$.

It is now worth noting that the gradient of the asymptote on the right hand side of the plot, which corresponds to the geometric regime, is equal to -1 . Therefore, from our dimensional analysis and the values in the last row of Table I, $m_{\delta_{G}}$ is again found to be 
equal to zero, since $m_{\delta_{G}}-1=-1$. This also confirms both that $m_{\Lambda_{G}}=-1$, demonstrated by our FE results, and also the independence of the attenuation coefficient to the frequency in the geometric regime, since $m_{f_{G}}$ must be approximately equal to 0 , from equation (11). A summary of the power relationships obtained from FE results, in all scattering regimes is shown in Table II.

TABLE II. Expected asymptotic power relationships, between the attenuation coefficient and the RMS height, correlation length and frequency. In this table, $q$ is the wavenumber, $\delta$ is the RMS height, $\Lambda$ is the correlation length and $f$ is the frequency.

\begin{tabular}{lccc}
\hline \hline Regime & Rayleigh & Stochastic & Geometric \\
\hline Limits & $q \delta<q \Lambda<1$ & $q \delta<1<q \Lambda$ & $1<q \delta \ll q \Lambda$ \\
\hline$\alpha(\delta, \Lambda, f)$ & $\delta^{1.77} \Lambda^{0.94} f^{3.77}$ & $\delta^{2.15} \Lambda^{-1.25} f^{1.94}$ & $\Lambda^{-0.83}$ \\
\hline \hline
\end{tabular}

Comparing Tables I and II, it is clear that there is good agreement in the asymptotic power relationship coefficient, across all scattering regimes. This has two implications firstly, we have managed to verify the well-established theory regarding scattering in the Rayleigh regime, both quantitatively and asymptotically. Secondly, our FE model was able to also verify the asymptotic in the stochastic and geometric regimes, confirming the applicability of assumptions from scattering ${ }^{12}$, to our study. 


\section{CONCLUSION}

A comprehensive study of the attenuation of Rayleigh waves from 2D statistically rough surfaces, using FE modelling has been presented. Three distinct scattering regimes have been identified from the literature - the Rayleigh (low frequency), stochastic (low to medium frequency) and geometric (high frequency) regimes. Analytical formulae, predicting attenuation values, have been derived in the past ${ }^{11}$, as well as asymptotic power relationships ${ }^{10,11}$ between $\alpha$ and $\delta, \Lambda$ and $f$.

Here, we attempted to validate the existing theory using FE analysis, and extend the results to regions where the theory is less established, or obtain results with a wider combination of $\delta, \Lambda$ and $f$ values. We have found good agreement between theory and FE results in all three regimes - in the Rayleigh and stochastic regimes, good agreement was found both quantitatively and asymptotically and the $f^{4}$ relationship between $\alpha$ and frequency in the Rayleigh regime was also observed. For the geometric regime, power relationships were derived by a combination of FE modelling and dimensional analysis.

The FE model's ability to follow the theory creates a plethora of useful implications. The theoretical formulae rely heavily on the $\omega_{2}$ function, which is a complicated function comprising multiple sub-functions, many of which have a different form depending on the region of interest, meaning that calculating $\omega_{2}$ is far from straightforward. The $\omega_{2}$ function's validity is also limited in terms of the roughness parameters for which it can produce results $(\delta / \Lambda<0.3)$ and its behaviour has not been studied extensively in the literature in the geometric regime. FE modelling removes the necessity to obtain this function, and allows for 
direct calculation of the attenuation coefficient. Additionally, the FE models can potentially be extended to regimes where the literature is more limited, such as the geometric regime, and can also simulate $\delta$ and $\Lambda$ parameters outside the theory's region of validity. Finally, the use of FE has provided a more unified approach to the study of rough surface scattering. In the literature, each scattering regime is largely studied in depth on its own, while the FE approach here has been able to verify the theory in all three regimes, by always implementing the same method.

Finally, it is worth noting that despite the results here being obtained from 2D simulations, they are still relevant for the 3D analytical formulae. The mathematical approach used to derive the $3 \mathrm{D}^{7}$ and $2 \mathrm{D}^{11}$ theory is analogous - therefore, the FE validation of the $2 \mathrm{D}$ theory in our study provides important insight for 3D theory, indicating that it will also hold true for FE simulations and experimental scenarios.

\section{ACKNOWLEDGMENTS}

Georgios Sarris is funded by the UK Research Centre in NDE, iCASE number 17000191, with contributions from Rolls-Royce Holdings plc and Jacobs Engineering Group Inc.. During this work Peter Huthwaite was part funded by the UK Engineering and Physical Sciences Research Council (EPSRC) Fellowship No. EP/M020207/1. Michael Lowe is partially sponsored by the EPSRC.

\section{REFERENCES}

${ }^{1}$ J. A. Ogilvy, Theory of Wave Scattering from Random Rough Surfaces (CRC Press, New 
York, 1991), pp. 1-37.

${ }^{2}$ H. Z. Maris, "Attenuation of ultrasonic surface waves by phonon viscosity and heat conduction," Phys. Rev. 188(3), 1308-1311 (1969).

${ }^{3}$ E. Salzmann, T. Plieninger, and K. Dransfeld, "Attenuation of elastic surface waves in quartz at frequencies of $316 \mathrm{MHz}$ and $1047 \mathrm{MHz}, "$ Appl. Phys. Lett. 13(14), 1308-1311 (1968).

${ }^{4}$ A. A. Maradudin and D. L. Mills, "The attenuation of Rayleigh surface waves by surface roughness," Ann. Phys. 10(1-2), 262-309 (1976).

${ }^{5}$ E. I. Urazakov and L. A. Fal'kovskii, "Propagation of a Rayleigh wave along a rough surface," Sov. Phys. - JETP 36(6), 1214-1216 (1972).

${ }^{6}$ J. W. S. Rayleigh, The Theory of Sound (Macmillan, London, 1877), pp. 1-370.

${ }^{7}$ A. G. Eguiluz and A. A. Maradudin, "Frequency shift and attenuation length of a Rayleigh wave due to surface roughness," Phys. Rev. B 28(2), 728-747 (1983).

${ }^{8} \mathrm{M}$. de Billy, G. Quentin, and E. Baron, "Attenuation measurements of an ultrasonic Rayleigh wave propagating along rough surfaces," J. Appl. Phys. 61, 2140-2145 (1987).

${ }^{9}$ V. V. Kosachev and Y. N. Gandurin, "Rayleigh wave dispersion and attenuation on a statistically rough free surface of a hexagonal crystal," Phys. Solid State 45(2), 391-399 (2003).

${ }^{10} \mathrm{~V}$. Chukov, "Rayleigh wave scattering by statistical arbitrary form roughness," Solid State Commun. 149(47-48), 2219-2224 (2009). 
${ }^{11} \mathrm{X}$. Huang and A. A. Maradudin, "Propagation of surface acoustic waves across random gratings," Phys. Rev. B 36(15), 7827-7839 (1987).

${ }^{12}$ A. Van Pamel, P. B. Nagy, and M. J. S. Lowe, "On the dimensionality of elastic wave scattering within heterogeneous media," J. Acoust. Soc. Am. 140(6), 4360-4366 (2016).

${ }^{13}$ I. M. Kaganova and A. A. Maradudin, "Surface acoustic waves on a polycrystalline substrate," Phys. Scr. T44, 104-112 (1992).

${ }^{14}$ V. V. Kosachev, Y. V. Lokhov, and V. N. Chukov, "Theory of attenuation of Rayleigh surface acoustic waves on a free randomly rough surface of a solid," Sov. Phys. - JETP 67(9), 1825-1830 (1988).

${ }^{15}$ F. E. Stanke and G. S. Kino, "A unified theory for elastic wave propagation in polycrystalline materials," J. Acoust. Soc. Am. 75(3), 665-681 (1984).

${ }^{16}$ J. A. Ogilvy, "Computer simulation of acoustic wave scattering from rough surfaces," J. Phys. D: Appl. Phys. 21(2), 260-267 (1988).

${ }^{17}$ J. A. Ogilvy and J. R. Foster, "Rough surfaces: Gaussian or exponential statistics?," J. Phys. D: Appl. Phys. 22(9), 1243-1251 (1989).

${ }^{18}$ J. Zhang, B. W. Drinkwater, and P. D. Wilcox, "Longitudinal wave scattering from rough crack-like defects," IEEE Trans. Ultrason., Ferroelectr., Freq. Control 58(10), 2171-2180 (2011).

${ }^{19}$ W. Choi, F. Shi, M. J. S. Lowe, E. A. Skelton, R. V. Craster, and W. L. Daniels, "Rough surface reconstruction of real surfaces for numerical simulations of ultrasonic wave scattering," NDT E Int. 98, 27-36 (2018). 
${ }^{20} \mathrm{~V}$. V. Kosachev and A. V. Shchergov, "Dispersion and attenuation of surface acoustic waves of various polarisations on a stress-free randomly rough surface of solid," Ann. Phys. 240(2), 225-265 (1995).

${ }^{21}$ S. G. Haslinger, M. J. S. Lowe, P. Huthwaite, C. R. V., and F. Shi, "Elastic shear wave scattering by randomly rough surfaces," J. Mech. Phys. Solids 137, 1-20 (2019).

${ }^{22}$ J. Zhang, B. W. Drinkwater, and P. D. Wilcox, "Effect of roughness on imaging and sizing rough crack-like defects using ultrasonic arrays," IEEE Trans. Ultrason., Ferroelectr., Freq. Control 59(5), 939-948 (2012).

${ }^{23}$ F. Shi, M. J. S. Lowe, and R. V. Craster, "Diffusely scattered and transmitted elastic waves by random rough solid-solid interfaces using an elastodynamic Kirchhoff approximation," Phys. Rev. B 95(21), 214305-1 - 214305-13 (2017).

${ }^{24}$ Rose, J. L., Ultrasonic Guided Waves in Solid Media (Cambridge University Press, New York, 2014).

${ }^{25} \mathrm{P}$. Huthwaite, "Accelerated finite element elastodynamic simulations using the GPU," J. Comput. Phys. 257(Part A), 687-707 (2014).

${ }^{26}$ S. G. Haslinger, M. J. S. Lowe, P. Huthwaite, R. V. Craster, and F. Shi, "Appraising Kirchhoff approximation theory for the scattering of elastic shear waves by randomly rough defects," J. Sound Vib. 460, 1-16 (2019).

${ }^{27}$ M. Huang, G. Sha, P. Huthwaite, S. I. Rokhlin, and M. J. S. Lowe, "Maximizing the accuracy of finite element simulation of elastic wave propagation in polycrystals," J. Acoust. Soc. Am. 148(40), 1890-1910 (2021). 
${ }^{28}$ A. A. E. Zimmermann, P. Huthwaite, and B. Pavlakovic, "High-resolution thickness maps of corrosion using SH1 guided wave tomography," Proc. R. Soc. A 477, 1-21 (2021).

${ }^{29}$ J. D. Achenbach, Wave Propagation in Elastic Solids (North Holland Publishing Company, Amsterdam, 1973), p. 192.

${ }^{30}$ M. B. Drozdz, "Efficient finite element modelling of ultrasound waves in elastic media," Ph.D. dissertation, Imperial College of Science Technology and Medicine, London, 2008.

${ }^{31}$ F. Shi, W. Choi, M. J. S. Lowe, E. A. Skelton, and R. V. Craster, "The validity of Kirchhoff theory for scattering of elastic waves from rough surfaces," Proc. R. Soc. A 471, $1-19(2015)$.

${ }^{32}$ B. B. Mandelbrot, The Fractal Geometry of Nature (W.H.Freeman \& Co Ltd, San Francisco, 1982), pp. 1-460.

${ }^{33}$ D. L. Jaggard, and Y. Kim, "Diffraction by band-limited fractal screens," J Opt Soc Am. 4(6), 1055-1062 (1987).

${ }^{34}$ J. B. Elliott, P. Huthwaite, M. J. S. Lowe, R. Phillips, and D. J. Duxbury, "Sizing Subwavelength Defects With Ultrasonic Imagery: An Assessment of Super-Resolution Imaging on Simulated Rough Defects," IEEE Trans. Ultrason., Ferroelectr., Freq. Control 66(10), 1634-1648 (2019).

${ }^{35}$ P. Rajagopal, M. B. Drozdz, E. A. Skelton, M. J. S. Lowe, and R. V. Craster, "On the use of absorbing layers to simulate the propagation of elastic waves in unbounded isotropic media using commercially available finite element packages," NDT E Int. 51, 30-40 (2012). 
${ }^{36}$ W. Hassan, M. Blodgett, and S. Bondok, "Numerical analysis of the Rayleigh wave dispersion due to surface roughness," in AIP Conference Proceedings (2004), Vol. 700, pp. 262-269.

${ }^{37}$ A. Boschetto, L. Bottini, and F. Veniali, "Surface roughness and radiusing of Ti6Al4V selective laser melting-manufactured parts conditioned by barrel finishing," Int. J. Adv. Manuf. Technol. 94, 2773-2790 (2018).

${ }^{38}$ V. Alfieri, P. Argenio, F. Caiazzo, and V. Segi, "Reduction of surface roughness by means of laser processing over additive manufacturing metal parts," Materials 10(1), 30-42 (2016).

${ }^{39}$ A. Boschetto, L. Bottini, and F. Veniali, "Roughness modeling of AlSi10Mg parts fabricated by selective laser melting," J. Mater. Process. Technol. 241, 154-163 (2017).

${ }^{40}$ J. R. Pettit, A. E. Walker, and M. J. S. Lowe, "Improved detection of rough defects for ultrasonic nondestructive evaluation inspections based on finite element modeling of elastic wave scattering," IEEE Trans. Ultrason., Ferroelectr., Freq. Control 62(10), 1797-1808 (2015). 


\section{TABLES}

TABLE I. Expected asymptotic power relationships, between the attenuation coefficient and the RMS height, correlation length and frequency. In this table, $q$ is the wavenumber, $\delta$ is the RMS height, $\Lambda$ is the correlation length and $f$ is the frequency.

\begin{tabular}{lccc}
\hline \hline Regime & Rayleigh & Stochastic & Geometric \\
\hline Limits & $q \delta<q \Lambda<1$ & $q \delta<1<q \Lambda$ & $1<q \delta \ll q \Lambda$ \\
\hline$\alpha(\delta, \Lambda, f)$ & $\delta^{2} \Lambda f^{4}$ & $\delta^{2} \Lambda^{-1} f^{2}$ & $\delta^{m_{\delta_{G}}} \Lambda^{-1-m_{\delta_{G}}}$ \\
\hline$\beta\left(\delta_{n}, \Lambda_{n}\right)$ & $\delta_{n} \Lambda_{n}^{2}$ & $\delta_{n}$ & $\delta_{n}^{m_{\delta_{G}}-1} \Lambda_{n}^{-m_{\delta_{G}}}$ \\
\hline \hline
\end{tabular}

TABLE II. Expected asymptotic power relationships, between the attenuation coefficient and the RMS height, correlation length and frequency. In this table, $q$ is the wavenumber, $\delta$ is the RMS height, $\Lambda$ is the correlation length and $f$ is the frequency.

\begin{tabular}{lccc}
\hline \hline Regime & Rayleigh & Stochastic & Geometric \\
\hline Limits & $q \delta<q \Lambda<1$ & $q \delta<1<q \Lambda$ & $1<q \delta \ll q \Lambda$ \\
\hline$\alpha(\delta, \Lambda, f)$ & $\delta^{1.77} \Lambda^{0.94} f^{3.77}$ & $\delta^{2.15} \Lambda^{-1.25} f^{1.94}$ & $\Lambda^{-0.83}$ \\
\hline \hline
\end{tabular}




\section{LIST OF FIGURE CAPTIONS}

Figure 1. (Colour online) Schematic of the FE model. A Tukey window is applied to the original rough surface, generated using the method described in subsection II A (in yellow), before it is inserted to form the lower boundary of the FE domain (in red). The scale of the rough surface is exaggerated for better visualisation.

Figure 2. (Colour online) Detail of the FE domain's meshing, after the Tukey-windowed rough surface has been applied to its lower boundary.

Figure 3. (Colour online) Example of a Rayleigh wave field, travelling in the positive $x$ direction created using the method described in this section. The colour scale in the figure represents the absolute magnitude of the displacement at each node. The Rayleigh wave's centre frequency is $6 \mathrm{MHz}$ and the rough surface has $\delta=25 \mu \mathrm{m}$ and $\Lambda=50 \mu \mathrm{m}$.

Figure 4. (Colour online) Variation of the attenuation coefficient, as the number of realisations increases, for three roughness scenarios, at $f=10 \mathrm{MHz}$.

Figure 5. (Colour online) Comparison of theoretical $\alpha$ values with FE results. The attenuation coefficient is plotted against the dimensionless quantity $q \Lambda$, which is analogous to plotting against frequency. The theoretical predictions of the model of ${ }^{11}$ are shown using the curve, and the FE results are plotted as $\times$.

Figure 6. (Colour online) FE results, relating to the Rayleigh regime. The figure shows the FE results, plotted as $\times$, and the line of best fit through them. The gradient of the best fit line, $\mathrm{m}$, is also shown on the figure. Values of the attenuation coefficient (either absolute 
or normalised) are plotted on the vertical axis, while the variable whose power relationship is investigated, is plotted on the horizontal axis.

Figure 7. (Colour online) FE results, relating to the stochastic regime. The figure shows the FE results, plotted as $\times$, and the line of best fit through them. The gradient of the best fit line, $\mathrm{m}$, is also shown on the figure. Values of the attenuation coefficient (either absolute or normalised) are plotted on the vertical axis, while the variable whose power relationship is investigated, is plotted on the horizontal axis.

Figure 8. (Colour online) $\alpha_{n}$ vs $\Lambda$ plot, geometric regime. The figure shows the FE results, plotted as $\times$, and the line of best fit through them. The gradient of the best fit line, $\mathrm{m}$, is also shown on the figure. Values of the attenuation coefficient (either absolute or normalised) are plotted on the vertical axis, while the variable whose power relationship is investigated, is plotted on the horizontal axis.

Figure 9. (Colour online) $\beta$ vs $\delta_{n}$ master plot. Here, generalised attenuation coefficient values are drawn for a large combination of frequencies and roughness parameters. The datapoints cover both the stochastic and geometric regions, where the above proposed ad hoc approximation predicts that $\beta \propto \delta_{n}$ and $\beta \propto \delta_{n}^{-1}$ respectively, with the transition occurring at the $\delta_{n}=1 / 2 \pi$ point. The Monte Carlo results with a fixed $\Lambda(=800 \mu \mathrm{m})$ are plotted as $\times$, the results with a common $f(=1 \mathrm{MHz})$ are plotted as $\circ$, and the results with a fixed $\delta(=200 \mu \mathrm{m})$ are plotted as $\diamond$. The dashed black lines show the asymptotic approximation for both scattering regimes, while the red dashed line indicated the transition point between them. 